\title{
Palladium-Catalyzed Cross-Coupling of 1,1-Diboronates
}

Category

Metal-Mediated

Synthesis

Key words

palladium

cross-coupling

$\mathrm{R}^{1}=\mathrm{Ar}$, Alk

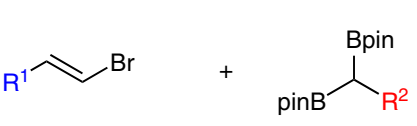

(0.75 equiv)

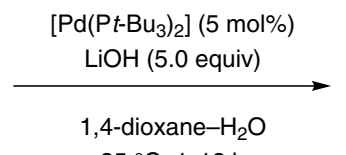

$25^{\circ} \mathrm{C}, 1-12 \mathrm{~h}$

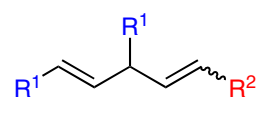

up to $94 \%$ yield

$\mathrm{R}^{2}=\mathrm{CH}_{2} \mathrm{Bn}$, i-Pr, $\mathrm{Cy}$

\section{Selected examples:}<smiles>CC(C)/C=C/C(/C=C/c1ccccc1)c1ccccc1</smiles>

$45 \%$ yield, $E / Z>20: 1$<smiles>CCCCC/C=C/C(/C=C/c1ccccc1)c1ccccc1</smiles>

$57 \%$ yield, $E / Z>20: 1$<smiles>C(=C/C(/C=C/c1ccccc1)c1ccccc1)\CCCc1ccccc1</smiles>

$80 \%$ yield, $E / Z=11: 1$

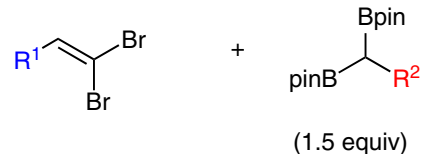

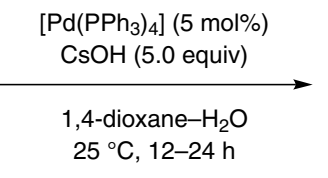

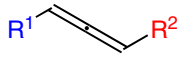

up to $79 \%$ yield

$\mathrm{R}^{1}=\mathrm{Ar}$, Alk

$\mathrm{R}^{2}=\mathrm{CH}_{2} \mathrm{Bn}$, Alk, $\mathrm{Cy}$

Selected examples:<smiles>Brc1ccc(C=CCCc2ccccc2)cc1</smiles>

$74 \%$ yield

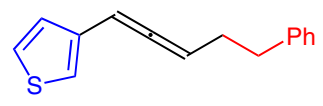

$79 \%$ yield

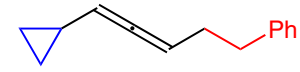

$76 \%$ yield
Significance: The authors demonstrate a palladium(0)-catalyzed reaction of 1,1-diboronates with substituted vinyl bromides or dibromoalkenes to give 1,4-dienes or allenes in good yields while showing good functional group tolerance.
Comment: When using 1,1-dibromoalkenes bearing a terminal alkynyl group as the substrate, the palladium(0)-catalyzed coupling described above can be followed by a Cul-catalyzed allenation with $N$-tosylhydrazones to give unsymmetrical diallenes. 